\title{
Chapter 1 Designing Biomimetic-Inspired Middleware for Anticipative Sensor-Actor Networks
}

\author{
Christopher Chiu, Zenon Chaczko
}

\begin{abstract}
Developing software environments for Sensor-Actor Networks (SANETS) is a promising research concern in systems engineering. Current concepts in software would adopt SANETS in a singular communications methodology, but the solution in this work is to take biological inspiration for the systems solution, thus the design of the system achieves a biomimetic construct as a result. SANETS are configurable for a variety of network structures and topologies, with the research aim in designing a network that is interactive and anticipatory to external and internal adaptations. Meanwhile, the eventbased changes are composed of scenarios, and the interactivity between external and internal actors. From the requirements of the end-user, the system must be responsive and interactive from the user perspective in real-time, while in addition offering the contextual data to make useful interpretation of systemic conditions from an anticipative view [2].
\end{abstract}

Key words: Agent-centric Simulation, Anticipative Systems, BiomimeticInspired Methods, Sensor-Actor Based Networks (Sanets), Wireless Sensor Networks (WSN)

\subsection{Introduction}

The essential basis of co-operating Sensor Actor Networks (SANETS) is the interactivity amongst wireless networking hardware and human actors, both actuator and sensor, that consistently exchange information for mutual benefit. The interactivity of SANETS with the environmental surrounds demands a service model that is distributed and robust for the end-user requirements [5]. The delivery and provisioning of a distributed, harmonized service model

Faculty of Engineering and I.T., University of Technology, Sydney, Australia

Email: \{Christopher.Chiu, Zenon.Chaczko\}@uts.edu.au 
is based on the coordination and collation of major tasks and requirements from heterogeneous data elements and resources [2].

The responsibilities of each SANET service can vary considerably, however the ultimate endpoint is to compliment the user's operational capability to predict and estimate developments that could occur amongst the SANET monitored environment. Developing the architecture for multi-agent concerns using SANET infrastructures ensures the elementary aspects of design is shaped during the engineering phase of development. The core aspects are highlighted below [7]:

- Anticipative: The design of the system must adapt to environments that evolve, because SANETS collectively sense and act upon the environment with the exception that they have a limited lifespan until replacement or repair is required. Additionally, technological adaptations will lead towards new types of devices joining the final network. The architecture of the system that consists of the multiple devices, both external and internal to the SANET system, is suitably designed as software-based agents [4]. The software agents capture the thresholds, specifications and behavior of the SANET devices throughout the systemic network, and agency interaction between each other and the behaviors that are observed.

- Interactive: Captures the dimensional views of the SANET-based space of problems, with additional components adding to the complete actuation and sensory functionality, depending on the conditions of the environment. The information is collected in a procedural manner for eventual postprocesses, and is executed at almost real-time for systemic efficiency. This is accomplished via the analysis of methodologies and the implementation of methods, by identifying heuristic-based approaches that is most suitable for the problem class that suits architectural designs for SANETs [6]. When the heuristic algorithm is sufficiently trained to detect local maxima and minima conditions, eventual computation of the data model is achieved.

- Biomimetic in Conception: Provisioning awareness of context to the architecture of the SANET, so it is capable for differences in the environment. The SANET network is configured for industrial, residential or commercial areas, with system complexity varying from heterogeneous to homogeneous domain concerns. The contextual variation is a core driver of the system architecture, while the system must be data focused - from the processing and aggregation of data as a complete structure [9]. This is achieved when one decouples the architecture with the heuristic software framework, to ensure the flexibility of the system accustoms to various methodologies of heuristics depending upon the space of the problem.

The results are expected to demonstrate the biomimetics-inspired facets of active heuristics combined with reinforcement-learned algorithms, optimally delivers a method for training systems in path navigation. Particularly, the construction of the SANET system as a multi-agent structure accomplished autonomic locality in perceiving senses, whilst ensuring global system objec- 
tives and targets. The robustness and adaptability of the system is done by constructing the SANET agency of service with a devoted heuristic algorithm, while the space of the problem is deconstructed throughout the simplification of concerned subset information.

The research accomplished is composed of the following structures: The essential theories and concepts of SANET architectural solutions are provided, with the following sections describing about the contribution of engineering research and the experimental data in tabular form. The complete observations and conclusions are examined in detail, with the incorporation of learning-reinforcement algorithms in conjunction with active-based functions of heuristics.

\subsection{SAnet Environments for Agency-based Architectures}

The main purpose of the experiment is to examine the Extended Spring Tensor Model (XSTEM) approach to analyze global perceptibility of the SANET domain. The main objective uses the open-based box experimental process, in order to contain the stages establishing the XSTEM-based model. The analytical approach of XSTEM assists in analyzing global interactivity, to view the SANET structure as motes driven by interactions throughout the externalized environment, because of the inherent distribution of interconnections with the SANET hardware motes.

The experimentation aims to evaluate the model of XSTEM to establish the magnitude and fluctuation of change within the SANET structure, so one can determine which model is suitable for distributed contexts in softwareagent middleware systems. The ability of the XSTEM model to be used in contexts other than bio-computing constructs is an interesting concern, while reliability and performance are considered for future experimental work.

The experimental purpose is to determine the capability of XSTEM models for globalized SANET domains, resulting from the regionalized effects that influence the networking structure as a whole. The XSTEM model calculates the multitude of network structure sets, so the research objective determines the model's capability to estimate future SANET network configurations resulting from environmental conditions occurring from an external standpoint. The experimental analysis will ultimately determine the final results, and reach an outcome to view the XSTEM model's ability to evaluate globalized concerns of SANETs, along with generalized experimental conclusions and research studies to consider in future.

The general procedure of the XSTEM-based experiment is elaborated as follows: 
(a) Determine the assumptions of the experiment for the Open-box Experiment approach;

(b) Evaluate the approach of the algorithm of the XSTEM-based model for SANET structures;

(c) Elaborate the method and procedure of the XSTEM-based model to observe globalized influences in the SANET structure;

(d) Cater for the final results of the XSTEM-based Open-box model; and

(e) Scrutinize the XSTEM-based model for SANET structures, along with future experimentation and examination.

\subsection{Experimental Approach and Procedure}

\subsubsection{Approach of the Extended STEM Algorithm}

The extension of the STEM approach is described in the algorithmic work of authors stated in [3, 9], by considering the SANET research work of [6] with the STEM algorithmic approach by [10]. The core steps of the XSTEM heuristic execution is focused on a reinforcement iteration in a singular fashion for feedback of sensor data from the externalized domain as summarized in Figure 1.1 on the facing page.

The coordinates of the sensor data supports the first order derivative values as follows in Figure 1.2 on the next page:

- Values of bonds supported by length of radius between adjacent motes $(r)$;

- Values of angles supported by angular bond amongst adjacent motes $(\theta)$;

- Values of dihedrals supported by angular dihedral amongst adjacent motes $(\phi)$; and

- Contacts of non-localized motes based from the non-adjacent radius of motes $\left(r_{i j}\right)$.

\subsubsection{Approach of the Mote Redistribution}

Afterwards, the results of the second order derivative determines the projected values of direction and magnitude. The remodeled sensory information then undergoes post-processing for mote redistribution:

- Random arrangement with Mersenne-Twister random generation;

- Matrix arrangement with Equidistant Lattice Structure; and

- Agglomeration arrangement with Particle Swarm Optimization. 


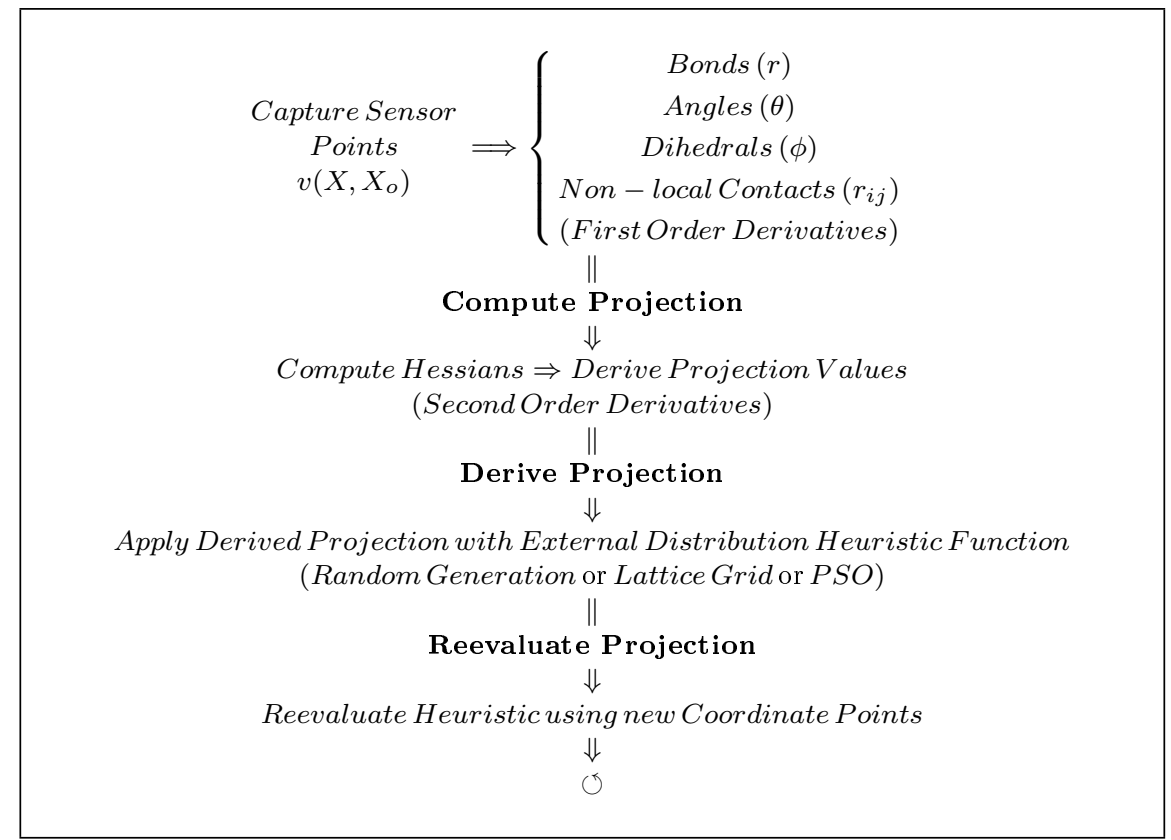

Fig. 1.1 Extended Stem Algorithm Process

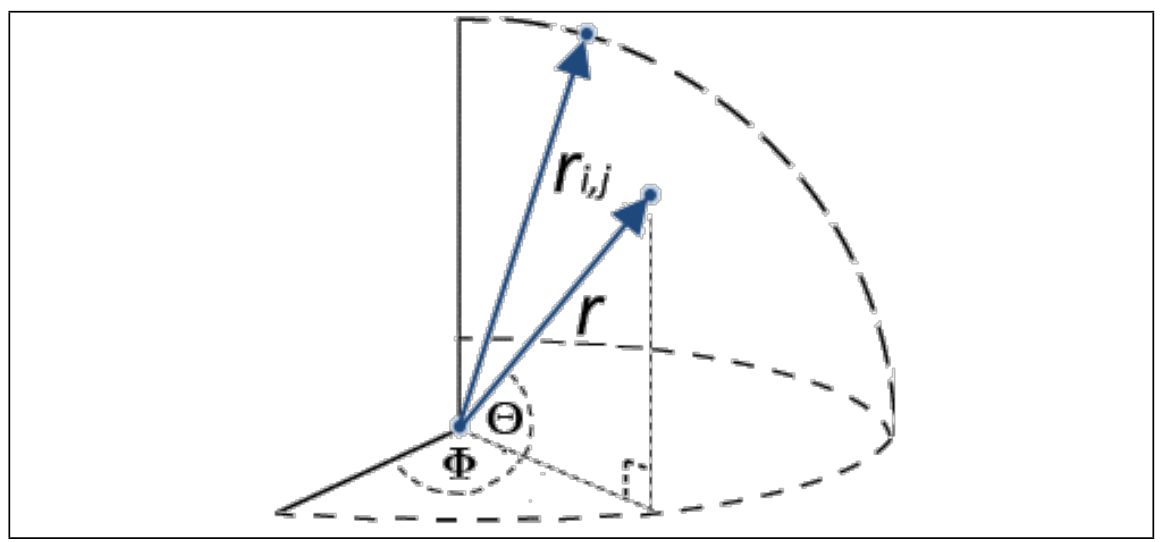

Fig. 1.2 Geometric Variables of the Sensor Coordinate Space 


\subsection{Procedure of Extended STEM Experiment}

The research procedure is conducted in accordance to the experimental approach as shown in Figure 1.3, with the main outcomes listed as follows:

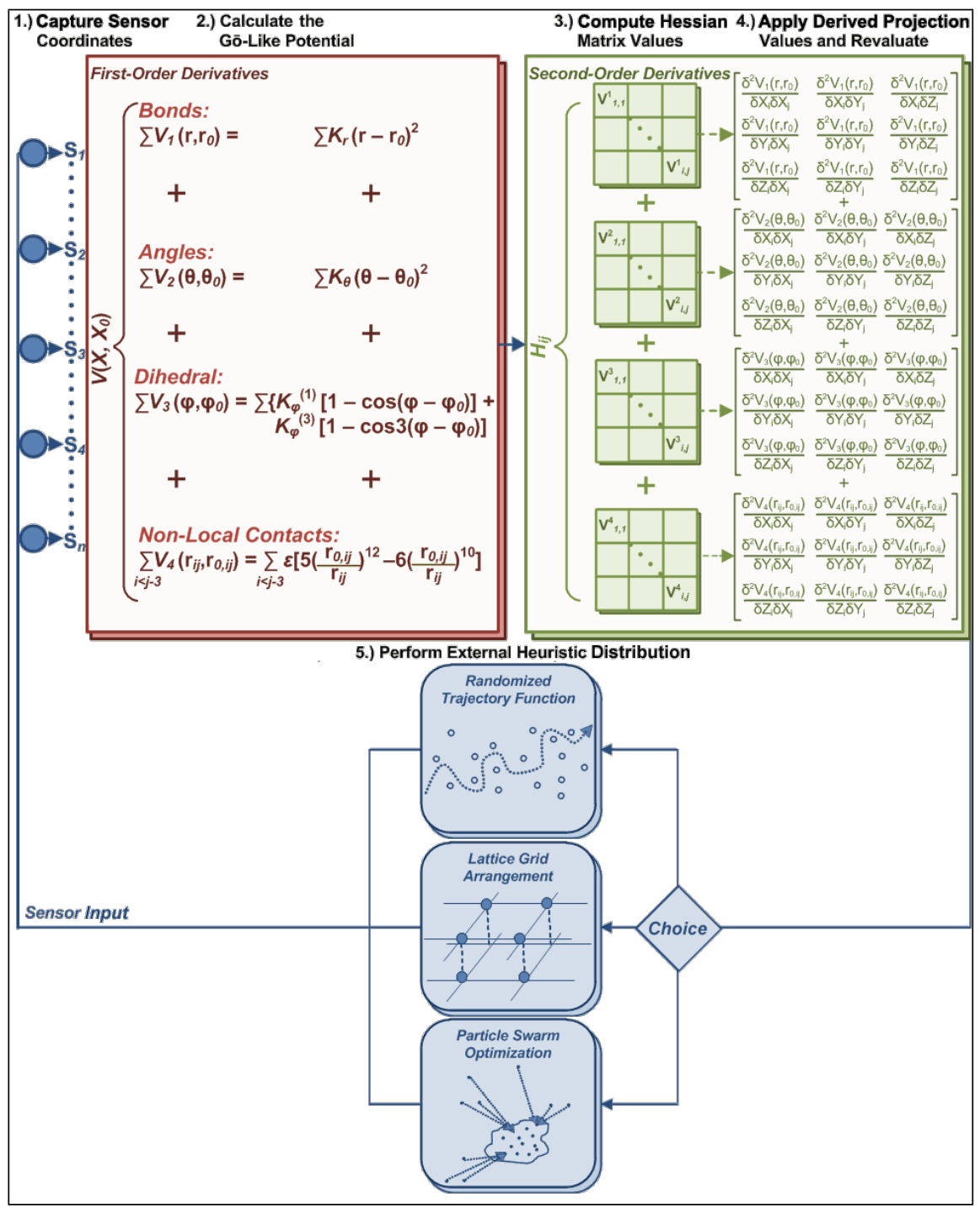

Fig. 1.3 Visual Block Diagram of Extended Sтем experiment 
- Aims: The aim of the XSTEm experiment is to ultimately determine the possibility of using a distributed SANET model for perception via global means, by evaluating against the heuristic of neural networks for optimization of the global optima. The method of Particle Swarm Optimization (PSO) is used to constitute the open-box experiments.

- Purpose: The purpose of the XSTEM experiment is to find out the effectiveness of XSTEM in the distributed sensor field, as the research study executed in a defined framework generates results that can be interpreted and analyzed from a SANET structure perspective.

- Limitations: The limits of the XSTEM experiment is that the model of the algorithm only considers the calculation of the projection of direction and magnitude from the SANET structure via global influences. The research study is operated on the MATLAB Toolkit by MathWorks Incorporated.

1. Whole amount of ' $n^{\prime}$ SANET motes is provided from the multi-agency input of data resources, in a 3-dimension network region of $50 \mathrm{~m}^{3}(\mathrm{~L} \times W \times H \Longrightarrow$ $5 m \times 5 m \times 2 m)$ in a 24 hour period. The general range from 25 to 200 motes an emulated rate of error for $5 \%$ to $10 \%$ of motes:
(a) 25 mote elements;
(e) 125 mote elements;
(b) 50 mote elements;
(f) 150 mote elements;
(c) 75 mote elements;
(g) 175 mote elements; and
(d) 100 mote elements;
(h) 200 mote elements.

The controlling specifiers of the research experiment are described below:

(i) Parameters of Data Input

The sensory information is gathered from the ZigBee network stack via a virtual serial-port interface consisting of a $9,600 \mathrm{bits} /$ second data rate. The program connects to the serial interface, formatting the information into a text-file of format Comma Separated/Delimited Value (Csv). The Matlab toolkit is executed to interpret the Csv text-file for the next process. The average sampling rate of the sensors is 2 reads/second.

(ii) Laboratory Structure

The laboratory structure is situated in the secure ICT Center located at the University of Technology, Sydney, Building 1, Floor 22. The laboratory is about $100 \mathrm{~m}$ above ground level, with air-conditioning set at $25^{\circ}$ Centigrade.

\section{(iii) Simulated Error Rate}

The Bit Error-Rate (BER) is generated using Sampling of Importance [11], with a probability of error that creates a randomized off-setting of the mote's position by $\pm 5 \%$ of the real position of the mote:

a. $5 \%$ Generated Ber: Creates in a random sample of $5 \%$ of all SANET motes providing irrelevant data and coordinate points. By means of Sampling of Importance, henceforth 500 samples of data is necessary to evaluate the error rate of maximum 5 motes in the information set with a 1:100 sample ratio. 
b. $10 \%$ Generated BER: Creates in a random sample of $10 \%$ of all SANET motes providing irrelevant data and coordinate points. By means of Sampling of Importance, henceforth 1,000 samples of data is necessary to evaluate the error rate of up to 10 motes in the information set with a 1:100 sample ratio.

2. The trajectory pathway is emulated in the SANET structure from a home geography of $x: 0 m, y: 0 m, z: 0 m$ to the destination geography of $x: 5 m, y: 5 m, z: 2 m$. The sensor events that motes will trigger proximity alerts only occur in a radius of $5 \mathrm{~cm}$ of the path. The distribution structure of the SANET network is described below:

\section{a. Distribution by Pseudo-Randomness}

The sensor motes are arranged randomly with the Mersenne-Twister approach. The seed is generated using the elapsed computer clock when the experiment takes place, with the value being the total seconds counted since January 1st, 1980.

\section{b. Distribution by Lattice-Grid Structure}

The sensor motes are equally distant amongst each other in a latticebased structure. The length among motes is arranged according to the total volume of the experimental testbed as stated in 1(ii).

\section{c. Distribution by Particle Swarm Optimization}

The sensor motes aggregate according to the algorithm of global optimization, comparing between the distribution approaches of 2(a) \& 2(b). Pso is used for this case study, as the effectiveness of the heuristic is examined in the following literature [8]. Pso values are as described:

(i) Total Heuristic Iterations $\quad 50$

(ii) Pso Factor of Inertia: $\quad 1.0$

(iii) Pso Corrective Factor: $\quad 2.0$

(iv) Pso Size of Swarm: SANET motes stated in 1(a-h)

3. The XStem approach is evaluated to determine the globalized direction and magnitude of SANET motes, using the values as defined by the following literature [1]:

(i) $K_{r}$ Length of Radius $=\quad 100 \varepsilon$

(ii) $K_{\theta}$ Angle of Bond $=\quad 20 \varepsilon$

(iii) $K_{\phi}^{(1)}$ Angle of Dihedral $=\quad \varepsilon$

(iv) $K_{\phi}^{(3)}$ Angle of Dihedral $=\quad 0.5 \varepsilon$

(v) $\varepsilon$ Value of Factor $=\quad 0.36$

Evaluating the capability of the XSTEM heuristic model for SANET structures is accomplished by the manner as stated:

a. Find the actual location of the SANET mote; 
b. Find the predicted location of the SANET mote using the XSTEm heuristic approach;

c. Determine the new location of the SANET mote;

d. Determine the location differential for the predicted and new location of the SANET mote, using the mote's actual location as a reference point.

4. The procedure is repeated for 100 cycles to determine the average success of identifying the predicted location for each SANET mote. The success of identification occurs where the estimated location is within $\pm 5 \%$ level of threshold of the predicted location:

a. The way to calculate the target of trajectory is achieved by calculating the spherical zone around the predicted location of the SANET mote. As

the distance between destination and home is $\sqrt{(5-0)^{2}+(5-0)^{2}+(2-0)^{2}}=$ $7.34 \mathrm{~m}$, the predicted location is estimated to be within $7.34 \times 5 \%=$ $\pm 36 \mathrm{~cm}$ of the mote's physical location.

\subsection{Results of the Extended STEM Experimentation}

\subsubsection{Analysis of the Extended Spring Tensor Analysis Model}

The experimental results of the XSTEM heuristic algorithm to estimate Random Data projection, shows that the increase of SANET motes from 25 to 200 devices results in a mean $16 \%$ betterment with $5 \%$ rate of error, and a mean $15 \%$ with $10 \%$ rate of error.

The trend in projection improves in a logarithmic fashion when the number of SANET mote agents increase, as the frequency of projection reaches $65 \%$ with $5 \%$ rate of error, and $60 \%$ with $10 \%$ rate of error. Increasing the overall rate of error from $5 \%$ to $10 \%$ results to a mean $3 \%$ decrease in estimating the projection.

\subsubsection{Lattice Grid Structure Formation}

The experimental results of the XSTEM heuristic algorithm to estimate Lattice Grid Structure projection, shows that the increase of SANET motes from 25 to 200 devices results in a mean $15 \%$ betterment with $5 \%$ rate of error, and a mean $14 \%$ with $10 \%$ rate of error.

The trend in projection improves in a logarithmic fashion when the number of SANET mote agents increase, as the frequency of projection reaches $66 \%$ with $5 \%$ rate of error, and $62 \%$ with $10 \%$ rate of error. Increasing the overall 
rate of error from $5 \%$ to $10 \%$ results to a mean $4 \%$ decrease in estimating the projection.

In comparison to Random Data arrangements, the Lattice Grid Structure operates in a more optimal fashion by a mean of $7 \%$ with $5 \%$ rate of error, and $6 \%$ with $10 \%$ rate of error. The differentiation in trend of projection for Random Data Structure and Lattice Grid Structure formation leads to an increase of $1 \%$ with $5 \%$ rate of error, and $2 \%$ with $10 \%$ rate of error.

\subsubsection{Particle Swarm Optimization Structure Formation}

The experimental results of the XSTEM heuristic algorithm to estimate Particle Swarm Optimization projection, shows that the increase of SANET motes from 25 to 200 devices results in a mean $16 \%$ betterment with $5 \%$ rate of error, and a mean $15 \%$ with $10 \%$ rate of error.

The trend in projection improves in a logarithmic fashion when the number of SANET mote agents increase, as the frequency of projection reaches $75 \%$ with $5 \%$ rate of error, and $70 \%$ with $10 \%$ rate of error. Increasing the overall rate of error from $5 \%$ to $10 \%$ results to a mean $5 \%$ decrease in estimating the projection.

In comparison to Lattice Grid arrangements, the Particle Swarm Optimization operates in a more optimal fashion by a mean of $8 \%$ with $5 \%$ rate of error, and $7 \%$ with $10 \%$ rate of error. The differentiation in trend of projection for Lattice Grid Structure and Particle Swarm Structure formation leads to an increase of $9 \%$ with $5 \%$ rate of error, and $8 \%$ with $10 \%$ rate of error.

\subsubsection{Comparison of Results for Extended Spring Tensor Model Heuristic}

The resulting improvement with the Extended Spring Tensor Model using Particle Swarm Optimization over the Random Data structure is elaborated below. By increasing the total population of SANET mote agents from 25 to 200 motes, a mean $10 \%$ betterment with $5 \%$ rate of error is recorded, and a mean $8.5 \%$ betterment with $10 \%$ rate of error is noted. Therefore, an overall mean betterment of $9 \%$ is observed.

The resulting improvement with the Extended Spring Tensor Model using Particle Swarm Optimization over the Lattice Grid structure results in the increase in SANET mote agents from 25 to 200 motes leads to a mean $6.5 \%$ betterment with $5 \%$ rate of error, and a mean $5.5 \%$ betterment with $10 \%$ rate of error. Therefore, an overall mean betterment of $6 \%$ is observed. 
Table 1.1 xStem Open-box Experimental Results

\begin{tabular}{|c|c|c|c|c|c|c|}
\hline \multirow{3}{*}{$\begin{array}{l}\text { No. of } \\
\text { Agent } \\
\text { Motes }\end{array}$} & \multirow{2}{*}{\multicolumn{2}{|c|}{$\begin{array}{c}\text { Random Structure } \\
\text { Rate of Error }\end{array}$}} & \multirow{2}{*}{\multicolumn{2}{|c|}{$\begin{array}{c}\text { Lattice-Grid Structure } \\
\text { Rate of Error }\end{array}$}} & \multicolumn{2}{|c|}{ Pso Structure } \\
\hline & & & & & \multicolumn{2}{|c|}{ Rate of Error } \\
\hline & \multicolumn{2}{|r|}{$10 \%$} & \multicolumn{2}{|c|}{$5 \% \quad 10 \%$} & $5 \%$ & $10 \%$ \\
\hline 25 & $16.891 \%$ & $16.223 \%$ & $18.353 \%$ & $17.562 \%$ & $20.980 \%$ & $19.920 \%$ \\
\hline 50 & $25.532 \%$ & $24.682 \%$ & $27.622 \%$ & $26.501 \%$ & $31.601 \%$ & $29.751 \%$ \\
\hline 75 & $35.881 \%$ & $34.581 \%$ & $39.021 \%$ & $37.312 \%$ & $44.862 \%$ & $42.562 \%$ \\
\hline 100 & $42.911 \%$ & $41.332 \%$ & $46.730 \%$ & $44.543 \%$ & $53.493 \%$ & $50.382 \%$ \\
\hline 125 & $45.632 \%$ & $44.210 \%$ & $49.423 \%$ & $46.991 \%$ & $56.592 \%$ & $53.581 \%$ \\
\hline 150 & $51.371 \%$ & $49.581 \%$ & $55.882 \%$ & $53.131 \%$ & $63.812 \%$ & $60.183 \%$ \\
\hline 175 & $54.041 \%$ & $52.172 \%$ & $58.792 \%$ & $56.433 \%$ & $67.201 \%$ & $63.502 \%$ \\
\hline 200 & $58.870 \%$ & $56.893 \%$ & $63.811 \%$ & $60.722 \%$ & $72.940 \%$ & $68.670 \%$ \\
\hline
\end{tabular}

Table 1.2 xStem with Pso Optimization vs. Randomized Distribution

\begin{tabular}{|c|c|c|}
\hline \multirow{2}{*}{$\begin{array}{c}\text { Number of } \\
\text { Total } \\
\text { Agent Motes }\end{array}$} & \multicolumn{2}{|c|}{ Pso versus Random Structure } \\
\cline { 2 - 3 } & $5 \%$ Randomized & 10\% Randomized \\
\hline 25 & $4.091 \%$ & $3.703 \%$ \\
\hline 50 & $6.073 \%$ & $5.072 \%$ \\
\hline 75 & $8.982 \%$ & $7.981 \%$ \\
\hline 100 & $10.580 \%$ & $9.040 \%$ \\
\hline 125 & $10.961 \%$ & $9.370 \%$ \\
\hline 150 & $12.441 \%$ & $10.601 \%$ \\
\hline 175 & $13.173 \%$ & $11.342 \%$ \\
\hline 200 & $14.072 \%$ & $11.783 \%$ \\
\hline
\end{tabular}

Table 1.3 xStem with Pso Optimization vs. Lattice-Grid Distribution

\begin{tabular}{|c|c|c|}
\hline \multirow{2}{*}{$\begin{array}{c}\text { Number of } \\
\text { Total } \\
\text { Agent Motes }\end{array}$} & \multicolumn{2}{|c|}{ Pso versus Lattice Grid Structure } \\
\cline { 2 - 3 } & $5 \%$ Randomized Error \\
\hline 25 & $2.631 \%$ & 10\% Randomized \\
\hline 50 & $3.982 \%$ & $2.361 \%$ \\
\hline 75 & $5.843 \%$ & $3.252 \%$ \\
\hline 100 & $6.762 \%$ & $5.253 \%$ \\
\hline 125 & $7.182 \%$ & $5.831 \%$ \\
\hline 150 & $7.931 \%$ & $6.591 \%$ \\
\hline 175 & $8.411 \%$ & $7.053 \%$ \\
\hline 200 & $9.132 \%$ & $7.072 \%$ \\
\hline
\end{tabular}




\subsection{Conclusion of Research Study}

The research study of the Extended Spring Tensor Model using the stated structure formations offers the analysis as follows, in order to establish the direction and magnitude of estimated change from a globalized Sensor-Actor Network perspective:

- Random Mote Structure with Extended STEM Model

XSTEM predicts the projection of randomly created sensor mote information by an average $40 \%$ for sensor mote populations ( 25 - 200) with $14 \%$ standard deviation. It is noted that the performance of projection is reduced by a mean of $4 \%$ when error-injected input data increases from $5 \%$ to $10 \%$; as such it is noted that the reliability of sensor information is important for performance optimization of the XSTEM algorithmic model.

- Lattice-Grid Structure with Extended STEM Model

XSTEM predicts the projection of equidistant lattice structures by an average of $43 \%$ for sensor mote populations (25 - 200) with $15 \%$ standard deviation. The experimental results demonstrate that XSTEM heuristic optimization of lattice structures offers projection estimation improvement over random data, as lattice structures incorporates geographic structural stability instead of randomized structures.

- Pso Distribution Structure with Extended STEM Model XStem predicts the project of Particle Swarm Optimization structures an average of $50 \%$ for sensor mote populations $(25-200)$ with $17 \%$ standard deviation. The XSTEM heuristic model in combination with Pso distribution structure offers projection estimation improvement over lattice and random structures, as Pso has the ability to be adaptable to variability in the externalized SANET network.

- Comparing XSTEM with Pso over Randomized \& Lattice Grid Structures

XSTEM projective capability of PSO distributions in comparison to random structures shows the predictive capability for Pso is increased by $9 \%$ in comparison to random structures, and is increased by $6 \%$ in comparison to lattice structures.

- Effect of Rates of Error

The estimation functionality of XSTEM is decreased when the rate of error being generated is increased from $5 \%$ to $10 \%$ of SANET motes. The level of difference is a mean reduction of $4 \%$ for random data structures, $5 \%$ for grid data structures and $6 \%$ for Pso structures.

The XSTEM heuristic results show for the distribution structure methodologies used in the research study, increasing SANET motes agents leads to an improvement in the projection rate estimation capability. The improvement in prediction capability results from the increasing interactivity, particularly amongst SANET motes being analyzed by the XSTEM heuristic algorithm. It is noted that the XSTEM predictive frequency is not in proportion to the 
population of sensor motes, with the limiting factor being the methodology of distribution for the SANET system structure.

In addition, the rate of error for the sensory information has an effect on the projection capability of the XSTEM heuristic, as observed in Table 1.1 on page 11. Throughout the distribution structures adopted, the XSTEM heuristic capability was lowered as the rate of error was raised from $5 \%$ to $10 \%$ of total SANET motes. Thus, the XSTEM heuristic must incorporate a filter for handling errors to reduce the sensory inputs of motes that malfunction, which ultimately improves the outcome of projection capability.

In conclusion, integrating XSTEM heuristics with PSO optimization approaches offers an optimum level of projection functionality over lattice-grid and randomized distribution structures, as Pso optimization behavior is more certain when compared to randomized distributions. The capability of XSTEM heuristics to project the mote's direction and magnitude of change demonstrates potential, especially when applying global tensor functions in SANETbased infrastructures.

\subsubsection{Future Task of Research Study}

The magnitude and projection of changes for SANET infrastructures can be extended beyond Euclidean metrics. The projection of the physical realm with non-Euclidean metrics will result in overlapping global with local dimensional domains. Additionally, the parametric constants and thresholds adopted for the XSTEM heuristic requires more study, such that the input constants accurately model the perceptions of SANET-based infrastructures.

The further work suggested for the XSTEM heuristic algorithm is applicable for contexts where SANET network spaces is utilized. Essentially, the physical realm can be integrated further standardized approaches, both in terms of data structures and protocols adopted. The advantages of using the approach means the controller can harmonize with externalized components that add value for the SANET network structure.

\section{References}

1. Cecilia Clementi, Hugh Nymeyer, Jose Nelson Onuchic. Topological and Energetic Factors: What Determines the Structural Details of the Transition State Ensemble and "En-route" Intermediates for Protein Folding? An Investigation for Small Globular Proteins. Journal of Molecular Biology, Elsevier Publishers, Volume 298, Issue 5, 2000.

2. Christopher Chiu, Zenon Chaczko. Design of Biomimetic Middleware for Anticipatory Sensor-Actor Network Systems. In Proceedings of the 2nd Asia-Pacific Conference on Computer-Aided System Engineering, APCASE 2014, 10-12 February 2014, South Kuta, Indonesia, page(s) 22-23, ISBN 978-0-9924518-0-6. 
3. Christopher Chiu, Zenon Chaczko. Enhancement of Surgical Training Practice with the Spring Tensor Heuristic Model. International Journal of Electronics and Telecommunications, Versita Publishers, Volume 59, Issue 3, 2013.

4. Dharma Agrawal, Bin Xie. Encyclopedia on Ad-Hoc and Ubiquitous Computing, Theory and Design of Ad-hoc Networks. World Scientific Publishers, Singapore, 2010.

5. Daniel Dubois. Introduction to Computing Anticipatory Systems. Journal of Computing Anticipatory Systems, Volume 314, Issue 2, 1998.

6. Holger Karl, Andreas Willig. Protocols and Architectures for Wireless Sensor Networks. Wiley Publishers, United States of America, 2007.

7. Leonid Perlovsky, Robert Kozma. Neurodynamics of Cognition and Consciousness. Springer-Verlag Publishers, Germany, 2007.

8. Maurice Clerc. Particle Swarm Optimization. Wiley Publishers, United States of America, 2006.

9. Robert Rosen. Dynamical Systems Theory in Biology. Wiley Publishers, United States of America, 1970.

10. Tu-Liang Lin, Guang Song. Generalized Spring Tensor Models for Dynamics and Conformation Changes. Structural Biology Journal, 2010.

11. Wheyming Tina Song, Wenchi Chiu, David Goldsman. Importance Sampling Techniques for Estimating the Bit Error Rate in Digital Communication Systems. 37th Winter Simulation Conference Proceedings, United States of America, 2005. 